\title{
Antagonism of Dexamethasone to Radiation Bystander Effect and Its Re-transmission
}

\author{
Zhan Hao ${ }^{1}$, Yao Ruoyu ${ }^{1}$, Zhang Xuguang, ${ }^{1, \text {, * }}$ \\ ${ }^{1}$ School of Clinical, Graduate School of Xuzhou Medical University, Xuzhou, China \\ ${ }^{2}$ Department of Radiotherapy, Xuzhou Cancer Hospital, Xuzhou, China
}

Email address:

zxgjh1992@qq.com (Zhang Xuguang)

${ }^{*}$ Corresponding author

To cite this article:

Zhan Hao, Yao Ruoyu, Zhang Xuguang. Antagonism of Dexamethasone to Radiation Bystander Effect and Its Re-transmission. Biochemistry and Molecular Biology. Vol. 4, No. 5, 2019, pp. 74-79. doi: 10.11648/j.bmb.20190405.12

Received: October 8, 2019; Accepted: November 8, 2019; Published: November 15, 2019

\begin{abstract}
Background: In clinical radiotherapy, in addition to the symptoms of radiation damage in the irradiated area, normal tissues can produce damage other than direct irradiation due to bystander effects. We often use dexamethasone to relieve symptoms, thereby protecting the surrounding normal tissues, and may also protect the tissues outside the target area. Objectives: To observe the antagonism of dexamethasone on radiation bystander effect and its Re-transmission. Materials and methods: After 6MV X-ray irradiation of rabbit, the plasma, the first generation conditioned medium, was prepared to treat rabbit lymphocyte, cultured for 24 hours, 24 hours later, lymphocytes were collected, and the second generation conditioned medium was prepared with the lymphocyte secretion to treat new rabbit lymphocyte, and the apoptotic rate of effecter lymphocyte was detected. Dexamethasone was used as a parallel treatment control in all experimental groups. Results: The first and second generations of conditioned medium can stimulate lymphocyte apoptosis. Dexamethasone could reduce the apoptotic level of rabbit lymphocytes treated with the first and second generation bystander conditioned medium from $21.087 \%$ to $14.957 \%, 28.695 \%$ to $20.205 \%$, respectively. Conclusion: Under certain conditions, dexamethasone can antagonize the injury of lymphocyte by radiation bystander effect, and can also antagonize the re-transmission of radiation bystander effect.
\end{abstract}

Keywords: Radiation Bystander Effect, Re-transmission, Dexamethasone

\section{Introduction}

Radiation bystander effect refers to the phenomenon that unirradiated cells exhibit the same or similar biological response as irradiated cells. In the clinical radiotherapy of tumor patients, radiation bystander effect is crucial, and it plays a role in both tumor tissues and normal tissues. In addition, radiation bystander effect may have a more profound impact on normal tissues. Our experimental studies have also confirmed that bystander effect can be re-transmitted, and bystander effector cells can release related substances, causing other cells to become effector cells. It is possible that bystander effects and their re-transmission play an important role in the side effects of radiation therapy. Clinically, various kinds of discomforts often occur during radiotherapy of patients. We often use dexamethasone to relieve symptoms, thereby protecting surrounding normal tissues, and may also protect tissues outside the target area. To this end, we have designed the following experimental ideas. The healthy rabbits were irradiated with X-rays, and the irradiated rabbit plasma was used to prepare a generation of conditioned medium, and the first-generation conditioned medium was used to treat the effector cells (rabbit blood lymphocytes), so that the effector cells showed a radiation bystander effect. Then, the normal culture solution is used to replace the first-generation conditioned medium, and the substance secreted by the effector cells is released into the new common culture solution, and then the culture solution is extracted to prepare the second-generation conditioned medium. Treatment of effector cells (rabbit lymphocytes) with a second-generation conditioned medium to see if dexamethasone antagonizes the first- and second-generation 
side effects? We conducted the following experiment.

\section{Materials and Methods}

\subsection{Experimental Animals}

New Zealand rabbits, male, about $3 \mathrm{Kg}$. Separation reagents and equipment are collected from conventional rabbit plasma and rabbit lymphocytes. Experimental animals were provided by the Animal Center of Xuzhou Medical University.

\subsection{Experimental Cells}

Rabbit blood lymphocytes were collected by conventional techniques.

\subsection{Cell Culture Techniques and Conventional (Unconditional) Cultures}

Rabbit lymphocyte unconditioned medium: Rabbit plasma was not irradiated (the treatment method was the same as that of irradiated rabbit plasma), and mixed with 1640 culture solution at $1: 2$, followed by addition of $1 \%$ by volume of double antibody (100 U/mL penicillin, $100 \mathrm{U} / \mathrm{mL}$ streptomycin).

\subsection{Conventional Methods for Collecting Rabbit Plasma and Isolating Rabbit Lymphocytes}

Separation reagents and equipment are collected from conventional rabbit plasma and rabbit lymphocytes.

\subsection{Conditioned Culture Solution Preparation}

\subsubsection{The First Generation Conditioned Medium Prepared} from Rabbit Plasma

i. Irradiation of New Zealand rabbits: New Zealand rabbit lower body was irradiated with 6MV X-ray, and the air volume was $20 \mathrm{~Gy}$ at one time.

ii. Rabbit plasma was filtered twice with a 0.22 um micropore filter to remove impurities.

iii. The filtered plasma is frozen at $-20^{\circ} \mathrm{C}$. After the plasma is coagulated, it is slowly dissolved at $4^{\circ} \mathrm{C}$, and the fibrin in plasma is precipitated and removed. After repeating 2 times, it is filtered once with a $0.22 \mu \mathrm{m}$ microporous filter. The first generation plasma was obtained.

iv. 5.1.4 The first generation plasma and 1640 medium were mixed in a volume of $1: 2$, and then $1 \%$ volume of the double antibody was added, which was a generation of conditioned medium for culturing rabbit lymphocytes.

v. The culture solution prepared from non-irradiated animal plasma is a control culture solution.

\subsubsection{Preparation of Second-generation Rabbit Plasma Conditioned Medium}

i. 5.2.1 The first generation of rabbit plasma conditioned medium + rabbit lymphocytes (first generation effector cells) were cultured for 24 hours, the conditioned medium was taken out, replaced with ordinary plasma unconditioned medium, and then the original lymphocytes (first generation effector cells) were cultured for 24 hours.

ii. Re-extract the culture solution, collect, centrifuge, and filter the cell debris with a filter to obtain the second-generation conditioned medium of rabbit plasma.

iii. The second-generation conditioned medium contains no (or rarely) irradiated material compared to the first-generation conditioned medium. The second-generation conditioned medium contains substances secreted by lymphocytes (first-generation effector cells) treated with a generation of conditioned medium as compared with ordinary unconditioned medium.

\subsection{Bystander Effect Damage Experiment}

Rabbit Lymphocyte Bystander Effect Damage Test

i. Effect of one-generation conditioned medium on rabbit lymphocytes.

1) Control group: 5 New Zealand rabbits, 3 parallel samples per animal. Lymphocytes + saline + normal plasma unconditioned medium were cultured for 24 hours.

Treatment group: 5 New Zealand rabbits corresponding to the previous one, and 3 parallel samples per animal. Lymphocytes (first generation effector cells) + normal saline + one generation plasma conditioned medium were cultured.

2) Lymphocytes were collected 24 hours after culture, and apoptosis was detected by flow cytometry.

ii. Effect of second-generation plasma conditioned medium on lymphocytes:

1) The control group and the treatment group were designed as above, and cultured in the second generation plasma conditioned medium.

2) Lymphocytes were collected 24 hours after culture, and apoptosis was detected by flow cytometry.

\subsection{Statistical Methods}

Data Entry After excel, logical proofreading, the database is generated. Data analysis was performed using spss 25.0 software. When the measurement data satisfies the normal distribution, the mean \pm standard deviation is used to represent the differences, and the differences between the two groups are compared using student's t-test.

\section{Results}

Culture normal lymphocytes from 5 New Zealand rabbits with one-generation conditioned medium, and detect apoptosis by Annexin V/PI cytometry. 
Table 1. Apoptosis rate of rabbit lymphocytes treated with rabbit plasma conditioned medium (\%).

\begin{tabular}{|c|c|c|c|c|c|}
\hline Animal label & Sample number & $\begin{array}{l}\text { Ordinary } \\
\text { plasma }\end{array}$ & $\begin{array}{l}\text { Ordinary plasma }+ \\
\text { DEX }\end{array}$ & $\begin{array}{l}\text { The first generation } \\
\text { medium }\end{array}$ & $\begin{array}{l}\text { The first generation } \\
\text { medium+ DEX }\end{array}$ \\
\hline & 1 & 8.80 & 6.05 & 24.40 & 20.30 \\
\hline \multirow[t]{3}{*}{1} & 2 & 8.20 & 6.50 & 22.70 & 14.75 \\
\hline & 3 & 8.10 & 6.55 & 23.20 & 13.20 \\
\hline & 1 & 8.45 & 6.30 & 21.80 & 15.00 \\
\hline \multirow[t]{3}{*}{2} & 2 & 7.50 & 6.90 & 21.70 & 14.00 \\
\hline & 3 & 7.20 & 6.85 & 19.95 & 15.80 \\
\hline & 1 & 7.75 & 5.85 & 22.10 & 13.75 \\
\hline \multirow[t]{3}{*}{3} & 2 & 8.25 & 6.45 & 19.70 & 15.15 \\
\hline & 3 & 8.35 & 6.45 & 21.15 & 14.40 \\
\hline & 1 & 8.75 & 6.00 & 21.00 & 15.65 \\
\hline \multirow[t]{3}{*}{4} & 2 & 6.40 & 5.75 & 21.05 & 15.10 \\
\hline & 3 & 7.80 & 5.90 & 19.35 & 14.15 \\
\hline & 1 & 8.35 & 4.85 & 19.0 & 16.45 \\
\hline \multirow[t]{2}{*}{5} & 2 & 8.15 & 6.75 & 20.25 & 14.25 \\
\hline & 3 & 8.80 & 6.65 & 18.95 & 12.40 \\
\hline average & & 8.057 & 6.253 & 21.087 & 14.957 \\
\hline Student's t-test $H$ value & & 1 & & 1 & \\
\hline
\end{tabular}

As can be seen from the above data, rabbit plasma (first-generation conditioned medium) irradiated with 20 Gy $\mathrm{X}$-rays can stimulate lymphocyte apoptosis. Dexamethasone can reduce the apoptotic rate of lymphocytes treated with a conditioned medium. At the same time, it can also reduce the apoptosis rate of lymphocytes in the ordinary plasma culture group.

Table 2. Apoptosis rate of rabbit lymphocytes treated with rabbit plasma second-generation conditioned medium (\%).

\begin{tabular}{|c|c|c|c|c|c|}
\hline Animal label & $\begin{array}{l}\text { Sample } \\
\text { number }\end{array}$ & $\begin{array}{l}\text { Ordinary } \\
\text { plasma }\end{array}$ & $\begin{array}{l}\text { Ordinary } \\
\text { plasma+DEX }\end{array}$ & $\begin{array}{l}\text { the second generation } \\
\text { medium }\end{array}$ & $\begin{array}{l}\text { the second generation } \\
\text { medium +DEX }\end{array}$ \\
\hline \multirow{3}{*}{1} & 1 & 13.42 & 15.57 & 33.16 & 21.40 \\
\hline & 2 & 12.27 & 14.33 & 29.85 & 20.35 \\
\hline & 3 & 12.03 & 12.07 & 30.94 & 20.61 \\
\hline \multirow{3}{*}{2} & 1 & 13.83 & 12.57 & 31.02 & 21.57 \\
\hline & 2 & 12.71 & 13.82 & 25.91 & 20.01 \\
\hline & 3 & 11.83 & 12.22 & 29.96 & 21.96 \\
\hline \multirow[t]{2}{*}{3} & 2 & 12.71 & 12.99 & 28.18 & 21.70 \\
\hline & 3 & 11.64 & 11.86 & 27.09 & 21.96 \\
\hline \multirow{3}{*}{4} & 1 & 11.72 & 12.60 & 25.62 & 19.69 \\
\hline & 2 & 12.12 & 11.83 & 30.01 & 20.81 \\
\hline & 3 & 12.88 & 12.45 & 29.01 & 18.35 \\
\hline \multirow[b]{2}{*}{5} & 1 & 11.39 & 10.55 & 27.16 & 19.32 \\
\hline & 2 & 12.35 & 11.74 & 28.69 & 19.22 \\
\hline average & & 12.377 & 12.53 & 28.695 & 20.205 \\
\hline Student's t-test $H$ value & & 0 & & 1 & \\
\hline
\end{tabular}

The above data indicate that the second-generation conditioned medium can stimulate lymphocyte apoptosis. Dexamethasone can reduce the apoptotic rate of lymphocytes treated with second-generation conditioned medium. However, dexamethasone did not reduce the apoptotic rate of lymphocytes in the normal plasma culture group, and the change was not obvious.

\section{Discussion}

In clinical radiotherapy, the radiation bystander effect is crucial, and its subjects include tumor tissue and normal tissue. From the clinical work point of view, we can find such a phenomenon, the side effects caused by radiotherapy may inhibit or damage the unirradiated tumor lesions, so this may become a new strategy for cancer treatment. For example, 1.
About $14 \%(4 / 28)$ of patients with renal cancer cells undergoing local radiotherapy, lesions of unexposed kidney cancer tissue are degraded, and the 4 patients have not received any other treatment before, indicating that kidney cancer cells Degenerative damage may occur through in vivo side effects of radiation therapy [1]. 2. In breast cancer patients treated with single radiotherapy as a tumor treatment, Konoeda had found $35.7 \%$ (15/42) patients side effects in metastatic lymph nodes, and 52.4\% (22/42) patients in histopathological examination Distal injury is found in the body, suggesting that the side effects in body are beneficial to tumor treatment [2]. 3. Camphausen $\mathrm{K}$ implanted mouse lung cancer tumors in the back and legs of C57BL/6 mice. After the tumor grew up, the tumor was locally irradiated and the tumor size of the distant leg was observed. It was found in wild type mice. The growth rate of the tumor in the 
irradiation group was significantly lower than that in the control group, which further proved that the side effect caused by radiotherapy is beneficial to the treatment of tumors in the unirradiated area [3].

Our previous work and current work, observed from the level of cell proliferation and apoptosis, radiation bystander effects can be transmitted between cells. Tumor cells, epithelial cells, and lymphocytes can all act as transmitters. They are side effector cells, and they are also transporting cells, and the second-generation side effects transmitted are quite powerful. Show that they are active participation, not just a "messenger." It can be understood that they respond to side effects at the cellular level [4]. In this experiment, it was observed that the serum of rabbits after 20Gy X-ray irradiation can stimulate cell apoptosis. The apoptosis rate of irradiated rabbit lymphocytes is much higher than that of normal cells, and the second-generation cell culture fluid can still promote cell apoptosis obviously, indicating that the radiation side effect exists and can be transmitted, and then dexamethasone is added. After that, the apoptotic rate of the cells was significantly lower than before, and the antagonism of dexamethasone on side effects and its retransmission was also shown.

In addition, radiation bystander effect is mainly a damage effect on normal tissues. 1. In the case of radiotherapy and chemoradiotherapy for patients with non-small cell lung cancer, Siva found DNA damage was observed in hair follicles that were not irradiated [5]. 2. Radiotherapy for non-brain parts causes multiple basal metabolism and persistent neuroinflammation in the brain. 3. Khan, Calveley irradiated the local lungs of rats, induced radiation side effects in the unexposed areas of the lungs, and DNA damage showed periodic changes $[6,7] .4$. Koturbash irradiated the skin of mice with a half-length, and after 96 hours of irradiation, the skin tissue in the adjacent area was always repaired for DNA damage [8]. 5. Koturbash, Tamminga irradiated the rat brain, the spleen showed significant epigenetic changes, and even maintained 7 months after irradiation; in the unirradiated reproductive organs such as testicular tissue DNA damage and changes in DNA methylation $[9,10]$.

Dexamethasone is a synthetic adrenal cortex hormone with anti-inflammatory, anti-viral, anti-immune, anti-shock and stress-relieving pharmacological effects. The relationship between dexamethasone and radiation is also included in tumor tissue and normal organizes. For the tumor tissue part, it tends to reduce radiosensitivity and protect normal tissues. For example, in the treatment of glioblastoma, dexamethasone can reduce the efficacy of radiotherapy. Aasland D found that radiotherapy could not activate the MGMT promoter of glioblastoma, while dexamethasone induced MGMT gene in cases of unmethylation of MGMT promoter, and may lead to further increase in temozolomide resistance, and reduce the effect of radiotherapy [11]. Luedi MM reported that dexamethasone reduced temozolomide-induced apoptosis in glioma stem cells and reduced radiation sensitivity. Camptothecin is a potential neutralizer for dexamethasone-mediated adverse reactions [12]. Ortega-Martínez S has the opposite report in the study of astrocytoma. Dexamethasone also plays a negative role in the treatment of prostate cancer [13]. Rane JK reported that dexamethasone reduced the expression of radiation-sensitive genes and decreased the sensitivity of prostate cancer cells. The glucocorticoid receptor inhibitor mifepristone increases gene expression and increases radiation sensitivity. For patients who received palliative radiotherapy for painful bone metastases, $40 \%$ experienced a brief increase in pain called a pain outbreak [14]. Furfari A reported that prophylactic dexamethasone reduced the incidence of pain episodes to $25 \%$. The substances associated with the dexamethasone reaction are derived from tumor extracellular matrix and cytoskeletal regulation-related genes. It is possible to predict the onset of pain and the dexamethasone response by genetic recognition for targeted preventive treatment [15].

The effect of dexamethasone on normal tissues is mainly protection. Weidenfeld $\mathrm{J}$ reported that animal base and stress-induced corticosterone levels increased at 9 and 20 weeks after whole brain irradiation, and negative feedback from glucocorticoids was impaired. It may be due to an abnormal reaction of the hippocampus-pituitary-adrenal cortex (HPA) axis, which may play an important role in regulating the feedback effect of glucocorticoids [16]. Surace $\mathrm{L}$ reports dexamethasone, which has limited complement activation and anti-tumor effects of the immune system during radiotherapy [17]. Alt $\mathrm{C}$ uses dexamethasone to protect microglia in the retina of ionized radiation mice and inhibit the infiltration of bone marrow mesenchymal stem cells [18].

In some cases, Fernanda $G$ suggests that irradiated tumor cells cause so-called immunogenic death: that is, cell death can effectively expose tumor antigens and elicit an anti-tumor immune response. When stimulated by antigen, $\mathrm{T}$ lymphocytes are transformed into lymphoblasts, and then differentiate into sensitized $\mathrm{T}$ lymphocytes, which are involved in cellular immunity, and their immune functions are mainly anti-intracellular infections. The antagonism of dexamethasone on the side effect of radiotherapy is likely to inhibit the transmission of cytokines secreted by lymphocytes, thereby antagonizing the transmission of side effects of radiotherapy, reducing the effect of side effects of radiotherapy on effector cells, thereby reducing the apoptosis of effector cells [19].

There are not many reports on the role of dexamethasone in the radiation side effect. Traditionally, dexamethasone (Dexamethasone), also known as dexamethasone and flumethalin, Dexamethasone is a synthetic glucocorticoid commonly used for the prevention and management of side effects in cancer patients undergoing chemotherapy. While it is effective as an anti-emetic and in preventing hypersensitivity reactions, dexamethasone depletes peripheral blood lymphocytes and impacts immune responses [20]. And that has the following effects: 1 . Anti-inflammatory effect: It can reduce and prevent the reaction of tissue to inflammation, thereby reducing the manifestation of 
inflammation. Hormones inhibit the accumulation of inflammatory cells, including macrophages and leukocytes, at sites of inflammation and inhibit phagocytosis, release of lysosomal enzymes, and synthesis and release of inflammatory chemical mediators. 2. Immunosuppressive effects: including preventing or inhibiting cell-mediated immune responses, delaying allergic reactions, reducing the number of $\mathrm{T}$ lymphocytes, monocytes, eosinophils, and reducing the binding capacity of immunoglobulins to cell surface receptors And inhibit the synthesis and release of interleukin, thereby reducing the conversion of $\mathrm{T}$ lymphocytes to lymphoblasts and reducing the expansion of the primary immune response. It can reduce the passage of immune complexes through the basement membrane and reduce the concentration of complement components and immunoglobulins. Robe PA reported that dexamethasone reduced gap junction-mediated intercellular communication in the bystander effect. No side-effects mediated by intercellular soluble substances have been retrieved in current studies [21]. In this experiment, the intercellular effect of soluble substances mediated by intercellular substances was observed. It was observed that dexamethasone can reduce the apoptosis level of rabbit lymphocytes treated with the firstand second-generation side effect conditioned medium, reaching one-third, and showed Strong protection. The overall experimental results are consistent with the relationship between dexamethasone and radiation. Specific to the radiation side effect level, no research report on glucocorticoids such as dexamethasone has been seen. More repeated trials are needed, and further research is needed at the molecular level to clarify the roles and mechanisms.

\section{Conclusion}

In clinical radiotherapy, radiation bystander effect is crucial, and it plays a role in both tumor tissues and normal tissues. Under certain conditions, dexamethasone can antagonize the injury of lymphocyte by radiation bystander effect, and can also antagonize the re-transmission of radiation bystander effect.

\section{Fund Project}

Natural Science Foundation of Jiangsu Province (BK20151156).

\section{References}

[1] Wersall PJ, Blomgren $\mathrm{H}$, Pisa $\mathrm{P}$, et a1. Regression of non-irradiated metastases after extracranial stereotactic radiotherapy in metastatic renal cell carcinoma [J]. ActaOncol, 2006, 45 (4): 493-497. DOI: 10.1080/02841860600604611.

[2] Konoeda K, Therapeutic efficacy of pre-operative radiotherapy on breast carcinoma: in special reference to its abscopal effecton metastatic lymph-nodes 1JI. Nihon Gan Chiryo Gakkai shi, 1990, 25 (61): 1204-1214.
[3] Camphausen K, Moses MA, Menard C, et al. Radiation abscopal antitumor effect is mediated throughp53 [J]. Cancer Res, 2003, 63 (8): 1990-1993.

[4] Yang S, Jing X, Shao W, et al. Radiation-Induced Bystander Effects in A549 Cells Exposed to 6 MV X-rays.[J]. Cell Biochemistry \& Biophysics, 2015, 72 (3): 1-6.

[5] Siva S, Lobachevsky P, Macmanus MP, et al. Radiotherapy for nonsmall cell lung cancer induces DNA damage response in both irradiated and out-of-field normal tissues [J]. Clin Cancer Res, 2016, 22 (19): 4817-4826. DOI: 10.1158/1078-O432.CCR-16-0138.

[6] Khan M A, Hill R P, Van Dyk J. Partial volume rat lung irradiation: an evaluation of early DNA damage [J]. Int $\mathrm{J}$ Radiat Oncol Biol Phys, 1998, 40 (2): 467-76.

[7] Calveley V L, Jelveh S, Langan A, etal. Genistein can mitigate the effect of radiation on rat lung tissue [J]. Radiat Res, 2010, 173 (5): 602-11.

[8] Koturbash I, Rugo R E, Hendricks C A, etal. Irradiation induces DNA damage and modulates epigenetic effectors in distant bystander tissue in vivo [J]. Oncogene, 2006, 25 (31): 4267-75.

[9] Koturbash I, Zemp FJ, Kutanzi K, Luzhna L, Loree J, Kolb B, Kovalchuk O. Sex-specific micro RNAome deregulation in the shielded bystander spleen of cranially exposed mice. [Cell Cycle. 2008 Jun 1; 7 (11): 1658-67. Epub 2008 Mar 23.

[10] Tamminga J, Koturbash I, Baker M, et al. Paternal cranial irradiation induces distant bystander DNA damage in the germline and leads to epigenetic alterations in the offspring [J] Cell Cycle, 2008, 7 (9): 1238-45.

[11] Aasland D, Reich TR, Tomicic MT, Switzeny OJ, Kaina B, Christmann M. Repair gene O6 -methylguanine-DNA methyltransferase is controlled by SP1 and up-regulated by glucocorticoids, but not by temozolomide and radiation. $\mathrm{J}$ Neurochem. 2018 Jan; 144 (2): 139-151. doi: 10.1111/jnc.14262. Epub 2018 Jan 1.

[12] Luedi MM, Singh SK, Mosley JC, Hatami M, Gumin J, Sulman EP, Lang FF, Stueber F, Zinn PO, Colen RR. A Dexamethasone-regulated Gene Signature Is Prognostic for Poor Survival in Glioblastoma Patients. J Neurosurg Anesthesiol. 2017 Jan; 29 (1): 46-58.

[13] Ortega-Martínez S. Dexamethasone acts as a radiosensitizer in three astrocytoma cell lines via oxidative stress. Redox Biol. 2015 Aug; 5: 388-97. doi: 10.1016/j.redox.2015.06.006. Epub 2015 Jun 23.

[14] Rane JK, Erb HH, Nappo G, Mann VM, Simms MS, Collins AT, Visakorpi T, Maitland NJ. Inhibition of the glucocorticoid receptor results in an enhanced miR-99a/100-mediated radiationresponse in stem-like cells from human prostate cancers. Oncotarget. 2016 Aug 9; 7 (32): 51965-51980. doi: 10.18632/oncotarget.10207.

[15] Furfari A, Wan BA, Ding K Wong A, Zhu L, Bezjak A, Wong R, Wilson CF, DeAngelis C, Azad A, Chow E, Charames GS. Genetic biomarkers associated with pain flare and dexamethasone response following palliative radiotherapy in patients with painful bone metastases. Ann Palliat Med. 2017 Dec; 6 (Suppl 2): S240-S247. doi: 10.21037/apm.2017.09.04. Epub 2017 Sep 20. 
[16] Weidenfeld J, Siegal T, Ovadia H. Delayed effects of brain irradiation--part 1: adrenocortical axis dysfunction and hippocampal damage in an adult rat model. Neuroimmunomodulation. 2013; 20 (1): 57-64. doi: 10.1159/000342522. Epub 2012 Nov 23.

[17] Surace L, Lysenko V, Fontana AO, Cecconi V, Janssen H, Bicvic A, Okoniewski M4, Pruschy M, Dummer R, Neefjes J, Knuth A, Gupta A, van den Broek M. Complement is a central mediator of radiotherapy-induced tumor-specific immunity and clinical response. Immunity. 2015 Apr 21; 42 (4): 767-77. doi: 10.1016/j.immuni.2015.03.009. Epub 2015 Apr 14.

[18] Alt C, Runnels JM, Mortensen LJ, Zaher W, Lin CP. In vivo imaging of microglia turnover in the mouse retina after ionizing radiation and dexamethasone treatment. Invest Ophthalmol Vis Sci. 2014 Jul 31; 55 (8): 5314-9. doi: 10.1167/iovs.14-14254.
[19] Fernanda G. Herrera, MD; Jean Bourhis, MD, PhD; George Coukos, MD, PhD. Radiotherapy Combination Opportunities Leveraging Immunity for the Next Oncology Practice [J]. CA CANCER J CLIN 2017; 67: 65-85.

[20] Wayne J. Aston, Danika E. Hope, Alistair M. Cook, Louis Boon, Ian Dick, Anna K. Nowak, Richard A. Lake, and W. Joost Lesterhuis. Dexamethasone differentially depletes tumour and peripheral blood lymphocytes and can impact the efficacy of chemotherapy/checkpoint blockade combination treatment. ONCOIMMUNOLOGY 2019, VOL. 8, NO. 11. 2019 July 4.

[21] Robe PA, Nguyen-Khac M, Jolois O, Rogister B, Merville MP, Bours V. Dexamethasone inhibits the HSV-tk/ganciclovir bystander effect in malignant glioma cells. BMC Cancer. 2005 Apr 2; 5: 32. 